\title{
Influência da classe social nas razões clínicas das perdas dentárias
}

\author{
Influence of social class on clinical reasons for tooth loss
}

\author{
Renata Cimões ${ }^{1}$ \\ Arnaldo de França Caldas Júnior ${ }^{2}$ \\ Eliane H elena Alvim de Souza ${ }^{2}$ \\ Estela Santos Gusmão ${ }^{3}$
}

${ }^{1}$ Departamento de Prótesee Cirurgia Buco Facial, UniversidadeFederal de Pernambuco.

Tr. Professor Artur de Sá 4, Cidade Universitária. 50740-521 Recife PE. renata.cimoes@globo.com ${ }^{2}$ Departamento de

Odontologia Social, FaculdadedeOdontologia, Universidade de

Pernambuco.

${ }^{3}$ Departamento deM edicina

Oral, Faculdadede

Odontologia, Universidade dePernambuco.
Abstract In order to determine the influence of social class on clinical reasons for tooth loss in M aceió, the Alagoas State capital in Northeast Brazil, a cross-sectional study was conducted of 466 adults whose ages ranged from 18 to 76 years. Socioeconomic and demographic data were collected through a questionnaire. Clinical examinations determined the reason for extracting teeth, recording the DMF-T of all patients, who were divided into social classes on the basis of the data gathered from the questionnaire: $54.1 \%$ of the subjects were female with a mean age of $33.73 \pm$ 13.68 years; $369(79.2 \%)$ of them had not completed their secondary education and 385 (82.6\%) had family incomes no more than four times the official minimum wage (mean $3.4 \pm 5.4$ ). The main reason for the loss of permanent teeth was caries. The patients presented an average of 16.59 \pm 6.96 decayed, lost or filled teeth and most of them $(219-47 \%)$ had a D M F-T of 11 to 20 teeth. A statistically significant difference was noted between reasons for tooth loss and social class $(P<0.001)$, leading to the conclusion that social class significantly influenced clinical reasons for tooth loss.

Key words Tooth loss, Social class, Epidemiology
Resumo Com o objetivo dedeterminar a influência da classe social nas razões clínicas das perdas dentárias na cidade de M aceió, Alagoas, foi realizado um estudo transversal que envolveu 466 indivíduos adultos, com idadeentre 18 a 76 anos. Foram coletados dados socioeconômicos e demográficos através de um formulário. 0 exame clínico determinou a razão pela qual o dente seria extraído e houve registro do CPO-D de todos os pacientes. Os pacientes foram incluídos em classes sociais com base nos dados col etados dos formulários. 54,1\% dos indivíduos que participaram da pesquisa eram do sexo feminino, a idade teve média de 33,73 \pm 13,68 anos, observou-se que 369 (79,2\%) não tinham completado o segundo grau e $385(82,6)$ apresentaram renda familiar de até quatro salários mínimos, com média de $3,4 \pm 5,4$. A principal razão dasperdas dos dentes permanentes foi a cárie dentária. Os pacientes apresentaram em média 16,59 \pm 6,96 dentes cariados, perdidos ou obturados, a maioria dos pacientes $219(47,0 \%)$ apresentaram CPO-D entre onze a vinte dentes. Observou-se associação estatisticamente significante entre a razão da perda e o grupo social a que pertencia o paciente $(P<0,001)$. Concluiu-se que a classe social influenciou significativamente na razão clínica da perda dentária.

Palavras-chave Perda dental, Classesocial, Epidemiologia 
Introdução

Os fatores socioeconômicos influenciam de forma significativa o processo saúde doença. 0 uso dos conceitos de classe social apresenta al gumas dificuldades de operacionalização, como o grande número de variáveis geradas, necessidade de amostra grande, existência de duas ou mais inserções para um mesmo indivíduo ${ }^{1}$. Os estudos epidemiológicos têm se utilizado pouco dos conceitos de classe social como uma categoria importante no processo de saúde-doença².

Nos estudos que abordam as razões sobre as perdas dentárias, observa-se que os indivíduos são classificados em classes, utilizando-seapenas como critérios a renda e escolaridade apresentadas por estes. M as outras questões devem ser abordadas para que os fatores biológicos (cárie, doença periodontal) que levam à extração dos dentes sejam distinguidos dos fatores sociais ${ }^{3}$. Alguns estudos evidenciam a cárie como a principal razão biológica que leva à extração dental, sendo influenciada pela condição socioeconômica determinada pela precária condição de saúde bucal e diminuição do acesso aos serviços ${ }^{4}$.

0 conhecimento das razões pelas quais os dentes permanentes são extraídos é necessário para organizar e efetivar estratégias adequadas para prevenção e tratamento das doenças bucais, pois com esse conhecimento será possível melhorar as condições de saúdebucal ${ }^{5}$. A mortalidade dental fornece informações sobrea condição de saúde bucal do ponto de vista da conservação dos dentes e do aproveitamento dos tratamentos preventivos e curativos realizados ${ }^{6}$. A manutenção da dentadura natural por toda a vida dos indivíduos deve ser o ideal para os profissionais de saúde bucal e de saúde pública. Observa-se que a perda de dentes causa transtornos psicossociais, como dificuldade de relacionamento, insegurança e outros de ordem sistêmica, como disfunções gastrointestinais, má nutrição?.

A razão da extração dental associada à classe social tem sido pouco avaliada nos estudos sobre perdas. 0 objetivo do estudo foi determinar a influência da classe social nas razões clínicas das perdas dentárias em adultos da cidade de Maceió, AL.

\section{Métodos}

O estudo foi realizado na cidade deM aceió, capital do estado de Alagoas, localizada na região Nordeste do Brasil, com uma população de
797.759 habitantes erenda per capita de $R \$ 2.283$. O tamanho da amostra foi calculado considerando a prevalência de perda por cárie de $50 \%$, nível designificância de $5 \%$, intervalo de confiança de $95 \%$, efoi usado um fator de correção igual a 1.2. Com isso, o número mínimo esperado foi de 461 pacientes.

Os participantes do estudo foram provenientes de centrosmunicipais de saúde (serviço público) e de clínicas de seguro saúde (serviço privado). Como requisitos, deveriam apresentar pelo menos um dente com indicação para extração, ter idadeigual ou superior a 18 anos enão recusar sua participação na pesquisa. 0 pesquisador responsável pela coleta de dados permanecia no local, previamente sorteado, e examinava os pacientes que tinham indicação para exodontia, voltava-se ao mesmo local até que o número pré estabelecido de pacientes fosse alcançado.

$\mathrm{N}$ a cidade de M aceió, existia, na época do estudo, 45 centros municipais de saúdee 22 clínicas de seguro saúde. Ficou estabelecido que trinta centros municipaise seteclínicas de seguro saúde tomariam parte no estudo, sendo obtido um total de 313 pacientes provenientes do serviço público e 153 do serviço privado, totalizando 466. A pós a seleção por sorteio, todos os centros receberam cartas informando sobre a proposta do estudo, não havendo nenhuma recusa.

Os dados socioeconômicos para determinação da classificação social foram obtidos através de um formulário preenchido antes do paciente ser examinado. O ptou-se pela classificação social de Lombardi et al. ${ }^{8}$, que se baseia na relação dos indivíduos com os bens de produção, discriminando as seguintes classes: burguesia, nova pequena burguesia, pequena burguesia tradicional, proletariado típico, proletariado não típico e subproletariado. As razões das perdas foram determinadas pelos critérios propostos por Kay \& Blinkhorn ${ }^{9}$, onde as extrações foram incluídas em oito categorias: cárie, doença periodontal, razões ortodônticas, razões pré-protéticas, trauma, pericoronarite, pedido do paciente e outras.

A pós a entrevista, os pacientes foram examinados em ambiente de consultório com luz artificial e tiveram a razão da extração determinada por um único examinador. 0 registro deCPO-D foi utilizado para verificar a reprodutibilidade, 0 dente que seria extraído foi considerado, no momento do exame, como perdido e dessa forma observou-se um valor de kappa de 0,836.

Para análise dos dados, foi utilizado o programa SPSS na versão 11.0. Foi usado o teste do Qui-quadrado para verificar a possível associa- 
ção entre as variáveis. Para a realização da análise bivariada que incluía a classe social, os indivíduos foram agrupados em três categorias. Grupo G1 - formado por indivíduos nas classes burguesia, nova pequena burguesia e pequena burguesia tradicional; Grupo G2 - formado pelas classes do proletariado típico e proletariado não típico e Grupo G3 - formado pelos indivíduos do subproletariado.

\section{Resultados}

A amostra estudada se constituiu na sua maioria por indivíduos do sexo feminino $(54,1 \%)$, a idade variou entre 18 a 76 anos, com média de 33,73 e desvio padrão de $\pm 13,68$ anos; em relação à escolaridade, observou-se que 369 (79,2\%) não tinham completado o segundo grau e $385(82,6)$ apresentaram renda familiar de até quatro salários mínimos, com média de 3,4 e desvio padrão de $\pm 5,4$ e mediana de dois salários mínimos mensais.

A principal razão das perdas dos dentes permanentes na população estudada foi a cárie, seguida pela doença periodontal. Em relação às classes, observou-sequea burguesia apresentou $100 \%$ deperda por cárie, mas deve-selevar em consideração que apenas um paciente foi incluído nesta categoria. 0 subproletariado apresentou perda por cárie na ordem de $75,9 \%$. A maior porcentagem de perda devido à doença periodontal foi observada na classe do proletariado típico, e as perdas por outras razões (razões ortodônticas, razões pré-protéticas, trauma, pericoronarite, pedido do pacientee outras) apresentaram-secom maior porcentagem entre os indivíduos da nova pequena burguesia (50\%). A Tabela 1 mostra a distribuição das razões das perdas em relação à classe social. De acordo com a metodologia adotada, 56 indivíduos não puderam ser classificados em nenhuma das classes.

0 registro do CPO-D revelou que em média os pacientes apresentaram $16,59 \pm 6,96$ dentes cariados, perdidos ou obturados, a mediana foi 17 , mínimo 2 e máximo 32, a maioria dos pacientes $219(47,0 \%)$ apresentaram CPO-D entre onze a vinte dentes. Ao relacionar o registro do CPO-D com as classes sociais, foi observado que os pacientes com registro de CPO-D entre onze a vinte dentes tiveram as mais altas freqüências nas classes proletariado típico, pequena burguesia tradicional, subproletariado eproletariado não típico, respectivamente (Tabela 2).

As razões das perdas foram categorizadas em perdas por cárie, doença periodontal e outras razões (ortodônticas, pré-protéticas, trauma, pericoronarite, pedido do paciente e outras), e estas foram relacionadas à classe social categorizada em grupos de classes. Observa-se que a perda por cárie aumentou, percentualmente, do grupo G1 para G3, a perda por doença periodontal oscilou de $10,8 \%$ no grupo $\mathrm{G} 2,12,3 \%$ no grupo $\mathrm{G} 3 \mathrm{e} 16,9 \%$ no grupo G2. Para perda por outras razões, a maior porcentagem foi observada para o grupo G3 ea menor no grupo G1.

A Tabela 3 mostra os dados relativos à essa associação, onde se observa que a classe social esteve estatisticamente associada à razão da perda dental.

A relação entre o CPO-D e os grupos sociaisé apresentada na Tabela 4, onde não foi observada associação significativa entre as variáveis.

Tabela 1. Distribuição das razões das perdas dentárias na cidade de Maceió-AL em relação à classe social, 2003.

\begin{tabular}{|c|c|c|c|c|c|c|c|c|c|}
\hline \multirow[b]{2}{*}{ Classe social } & \multicolumn{8}{|c|}{ Razão da perda } & \multirow[b]{2}{*}{$\begin{array}{c}\text { Total } \\
\text { N (\%) }\end{array}$} \\
\hline & $\begin{array}{l}\text { Cárie } \\
\text { N (\%) }\end{array}$ & $\begin{array}{l}\text { D.P. }{ }^{1} \\
\text { N }(\%)\end{array}$ & $\begin{array}{l}\text { P.Próte }{ }^{2} \\
\text { N (\%) }\end{array}$ & $\begin{array}{l}\text { Orto }^{3} \\
\mathrm{~N}(\%)\end{array}$ & $\begin{array}{c}\text { Trauma } \\
\text { N (\%) }\end{array}$ & $\begin{array}{l}\text { Pedido } 4 \\
\text { N (\%) }\end{array}$ & $\begin{array}{c}\text { Pericoronarite } \\
\text { N (\%) }\end{array}$ & $\begin{array}{l}\text { Outras } \\
\mathrm{N}(\%)\end{array}$ & \\
\hline N classificável & $35(59,3)$ & $3(5,1)$ & $1(1,7)$ & $14(23,7)$ & 0 & $6(10,2)$ & 0 & 0 & $59(100)$ \\
\hline Burguesia & $1(100)$ & 0 & 0 & 0 & & 0 & 0 & 0 & $1(100)$ \\
\hline N ova pequena burguesia & $7(43,8)$ & $1(6,2)$ & $2(12,6)$ & $4(25,0)$ & $1(6,2)$ & $1(6,2)$ & 0 & 0 & $16(100)$ \\
\hline Pequena burguesia trad. & $11(55,0)$ & $3(15,0)$ & $1(5,0)$ & $3(15,0)$ & 0 & $2(10,0)$ & 0 & 0 & $20(100)$ \\
\hline Proletariado não típico & $96(55,2)$ & $27(15,5)$ & $6(3,5)$ & $33(18,9)$ & $1(0,6)$ & $7(4,0)$ & $3(1,7)$ & $1(0,6)$ & $174(100)$ \\
\hline Proletariado típico & $3(33,3)$ & $4(44,5)$ & 0 & $1(11,1)$ & 0 & $1(11,1)$ & 0 & 0 & $9(100)$ \\
\hline Subproletariado & $142(75,9)$ & $23(12,3)$ & $5(2,7)$ & $1(0,5)$ & 0 & $15(8,1)$ & $1(0,5)$ & 0 & $187(100)$ \\
\hline Total & $295(63,3)$ & $61(13,1)$ & 15 & 56 & 2 & 32 & 4 & 1 & 466 \\
\hline
\end{tabular}

1- D.P. - doença periodontal; 2 - P. Prote - pré-protetico; 3 - Orto - ortodôntico; 4 - Pedido - pedido do paciente. 
Tabela 2. Distribuição do registro do CPO-D em relação à classe social na cidade de Maceió-AL, 2003.

\begin{tabular}{lcccc}
\hline & \multicolumn{4}{c}{ CPO-D } \\
\cline { 2 - 4 } Classe social & $1-10$ & $11-20$ & + de 20 & Total \\
& $\mathrm{N}(\%)$ & $\mathrm{N}(\%)$ & $\mathrm{N}(\%)$ & $59(100)$ \\
\hline Não classificável & $19(32,3)$ & $31(52,5)$ & $9(15,2)$ & $1(100)$ \\
Burguesia & 0 & 0 & $1(100)$ & $16(100)$ \\
Nova pequena burguesia & $1(6,3)$ & $7(43,7)$ & $8(50,0)$ & $20(100)$ \\
P burguesia tradicional & $4(20,0)$ & $10(50,0)$ & $6(30,0)$ & $174(100)$ \\
Proletariado não típico & $37(21,3)$ & $77(44,2)$ & $60(34,5)$ & $9(100)$ \\
Proletariado típico & 0 & $7(77,8)$ & $2(22,2)$ & $187(100)$ \\
Subproletariado & $37(19,8)$ & $87(46,5)$ & $63(33,7)$ & $466(100)$ \\
Total & $98(21,0)$ & $219(47,0)$ & $149(32,0)$ & \\
\hline
\end{tabular}

Tabela 3. Distribuição das razões das perdas na cidade de Maceió-AL em relação aos grupos sociais, 2003.

\begin{tabular}{|c|c|c|c|c|c|c|c|c|c|}
\hline \multirow{3}{*}{ Grupo social (2) } & \multicolumn{6}{|c|}{ Razão da perda } & & & \multirow{3}{*}{ Valor de $\mathrm{P}^{(1}$} \\
\hline & \multicolumn{2}{|c|}{ Cárie } & \multicolumn{2}{|c|}{ Doença periodontal } & \multicolumn{2}{|c|}{ Outras } & \multicolumn{2}{|c|}{ Total } & \\
\hline & $\mathrm{N}$ & $\%$ & $\mathrm{~N}$ & $\%$ & $\mathrm{~N}$ & $\%$ & $\mathrm{~N}$ & $\%$ & \\
\hline G1 & 19 & 51,4 & 4 & 10,8 & 14 & 37,8 & 37 & 100,0 & \\
\hline $\mathrm{G} 2$ & 99 & 54,1 & 31 & 16,9 & 53 & 29,0 & 183 & 100,0 & $P<0,001^{*}$ \\
\hline G3 & 142 & 75,9 & 23 & 12,3 & 22 & 11,8 & 187 & 100,0 & \\
\hline Grupo total(3) & 260 & 63,9 & 58 & 14,3 & 89 & 21,9 & 407 & 100,0 & \\
\hline
\end{tabular}

* - Associação significante ao nível de $5,0 \%$.

(1) Através do teste Qui-quadrado; (2) Grupo G1 - formado por indivíduos nas classes burguesia, nova pequena burguesiae pequena burguesia tradicional; Grupo G2 - formado pelas classes do proletariado típico eproletariado não típico e Grupo G3 formado pelos indivíduos do subproletariado; (3) 59 pesqui sados não foram classificados segundo a metodologia empregada.

Tabela 4. Distribuição do índice CPO-D em relação aos grupos sociais, M aceió-AL, 2003.

\begin{tabular}{|c|c|c|c|c|c|c|c|c|c|}
\hline \multirow{3}{*}{ Grupo (1) } & \multicolumn{6}{|c|}{ CPO-D } & & & \multirow{3}{*}{ Valor de $\mathrm{P}^{(1)}$} \\
\hline & \multicolumn{2}{|c|}{$1-10$} & \multicolumn{2}{|c|}{$11-20$} & \multicolumn{2}{|c|}{+ de 20} & \multicolumn{2}{|c|}{ Total } & \\
\hline & $\mathrm{N}$ & $\%$ & $\mathrm{~N}$ & $\%$ & $\mathrm{~N}$ & $\%$ & $\mathrm{~N}$ & $\%$ & \\
\hline G1 & 5 & 13,5 & 17 & 46,0 & 15 & 40,5 & 37 & 100 & \\
\hline $\mathrm{G} 2$ & 37 & 20,2 & 84 & 45,9 & 62 & 33,9 & 183 & 100 & 0,073 \\
\hline G3 & 37 & 19,8 & 87 & 46,5 & 63 & 33,7 & 187 & 100 & \\
\hline Total (2) & 79 & 19,4 & 188 & 46,2 & 140 & 34,4 & 407 & 100 & \\
\hline
\end{tabular}

*- Através do teste Qui-quadrado.

(1) Grupo G1 - formado por indivíduos nas classes burguesia, nova pequena burguesia e pequena burguesia tradicional; Grupo G2 - formado pelas classes do proletariado típico eproletariado não típico e Grupo G3 - formado pelos indivíduos do subproletariado; (2) 59 pesquisados, sendo 19 do serviço privado e 40 do serviço público, não foram classificados segundo a metodologia empregada. 


\section{Discussão}

0 presente estudo apresenta al gumas limitações e as constatações não podem ser consideradas para população em geral. 0 critério de classe social aqui adotado não obteve classificação para todos os pacientes que tinham indicação para realização de pelo menos uma exodontia; talvez o critério que utiliza a posse de bens de consumo fosse mais indicado para este tipo de pesquisa.

A pesar do conhecimento sobre a importância dos fatores socioeconômicos na determinação dos processos saúde-doença, nos estudos sobre razões de perdas dentárias, esse aspecto não tem sido abordado com freqüência. Os índices de perda dental estão para O dontologia como os índices de mortalidade estão para M edicina, sendo necessário o conhecimento desses dados para auxiliar no planejamento dos serviços de saúde ${ }^{10}$. No Brasil, poucos estudos foram realizados para se obter informações sobre as razões das extrações dos dentes permanentes e, mesmo sendo observada uma queda nos índices de cárie para crianças, ainda é evidente que os adultos perdem muitos dentes devido à cárie dentária. No presente estudo, ficou evidente a grande quantidade de dentes extraídos por esta razão, assim como em outros estudos realizados no Brasil, onde a cárie predominou como razão primária ${ }^{3,10,11,12,13}$.

Para a cidade de M aceió, a perda por cárie foi na ordem de 63,3\%; esse dado é discordante das demais cidades brasileiras, onde já foram determinadas as prevalências de razões de perdas. Para a cidade do Recife, a cárie representou $70,3 \%$ de todas as perdas ${ }^{10}$; em Belo Horizonte, em dois estudos realizados, a cárie representou $61 \%{ }^{14} \mathrm{e}$ $42 \%{ }^{11}$; para Campo Grande, a porcentagem de perda por cárie foi de $58,5 \%{ }^{15}$. Em relação às perdas por doença periodontal, observou-se, tanto neste estudo quanto nos citados anteriormente, que foi caracterizada como a segunda maior prevalência encontrada. N esse estudo, ficou evidenciado uma pequena porcentagem de perda por doença periodontal, fato também demonstrado em outros estudos. Em Belo H orizonte foi onde se evidenciou a maior porcentagem de perda por doença periodontal dentre os estudos já realizados no Brasil ${ }^{16}$.

No presente estudo, ficou evidenciado que a classe social teve influência sobre as razões das perdas; os pacientes incluídos no grupo social 1 (G1) , que representava a burguesia, nova pequena burguesia e pequena burguesia tradicional, tiveram percentualmente menos perda por cárie quando comparados aos grupos G2 e G3. Para as perdas devido a outras razões, ficou demonstrado que o grupo G1 apresentou percentualmente mais perda; isso se deve ao fato de neste item estarem incluídas as indicações ortodônticas e protéticas. Em outros estudos, ficou evidenciado que a classe social baixa determina a perda dentária por razões biológicas (cárie, doença periodontal), pois para esses indivíduos não há recursos para que a saúde seja restabelecida, perdem mais dentes por cárie e apresentam maior número de dentes perdidos ${ }^{3,11}$, o quenão ocorre para indivíduos de classe social el evada, onde a indicação para exodontia pode ser revertida tanto para tratamentos restauradores como os protéticos.

Para 59 indivíduos, sendo dezenove provenientes de serviço privado e quarenta de serviço público, não foi possível a categorização segundo a classe social. Estes pacientes foram considerados não classificáveis por não apresentarem atividaderemunerada para quefossem incluídosnos processos de produção, sen do exemplos estudantes e donas-de-casa. A classificação social adotada apresenta algumas limitações quanto a este aspecto, mas Lombardi et al. ${ }^{8}$, ao comparar esta classificação com a desenvolvida por Barros ${ }^{16}$, demonstraram vantagens no que diz respeito a não superestimar a classe do subproletariado.

A maioria dos pacientesque participaram desta pesquisa tinham registro CPO-D entre onze a vinte dentes. Na faixa que apresentou CPO-D de zero a dez dentes, houve maior porcentagem de pacientesquenão puderam ser classificados. Para a faixa entre onze a vinte dentes, a maior porcentagem foi observada na classe do proletariado típico; já para a faixa acima de vinte dentes no registro CPO-D, a burguesia apresentou apenas um pacientequeficou inserido nessa categoria, seguido pela classe da nova pequena burguesia.

A classe social teve influência significativa na determinação das razões clínicas das perdas dentárias da população avaliada na cidade de Maceió, sendo que as classes que formaram o G1 (burguesia, nova pequena burguesia e pequena burguesia tradicional) tiveram menos perda por cárie e mais por outras razões (trauma, pericoronarite, indicações ortodônticas e protéticas, pedido do paciente e outras).

Os estudos epidemiológicos que evidenciam as razões das extrações dentárias devem adotar os critérios de determinação de classe social para que seja determinada a influência desta na razão clínica que leva os dentes a serem extraídos. 


\section{Colaboradores}

R Cimões trabalhou em todas as fases do estudo desde a coleta de dados até a elaboração do artigo. AF Caldas Júnior trabalhou no delineamento da metodologia eanálise dos dados. EHA Souza trabalhou no delineamento da metodologia. ES Gusmão trabalhou na concepção e na redação final do artigo.

\section{Agradecimentos}

Esta pesquisa foi apoiada pela CAPES, Brasil.

\section{Referências}

1. Solla JJSP. Problemas e limites da utilização do conceito de classe social em investigações epidemiológicas: uma revisão crítica da literatura. Cad Saúde Pública 1996; 12(2):207-216.

2. Solla JJSP. Diferenças nas propostas de operacionalização do conceito de classe social empregadas em estudos epidemiológicos. Cad Saúde Pública 1996; 12(3):329-337.

3. Guimarães $M M M, M \operatorname{arcos} B$. Expectativa de perda de dente em diferentes classes sociais. Rev CROM G 1996; 2(1):16-20.

4. Bailit HL, Braun R, Maryniuk GA, Camp P. Is periodontal disease the primary cause of tooth extraction in adults? J Am Dent Assoc 1987; 114(1):40-45.

5. Chestnutt IG, Binnie VI, Taylor M M. Reasons for tooth extraction in Scotland. J Dent 2000; 28:295-297.

6. Angelillo IF, Nobile CGA, Pavia M. Survey of reasons for extraction of permanent teeth in Italy. Community Dent Oral Epidemiol 1996; 24:336-340.

7. Sheiham A, Steele JG, Marcenes W, Lowe C, Finch $\mathrm{S}$, Bates $\mathrm{CJ}$ et al. The relationship among dental status, nutrient intake, and nutritional status in older people. J Dent Res2001; 80(2):408-415.

8. Lombardi C, Bronfman M, Facchini LA, Victora CG, Barros FC, Béria JU et al. O peracionalização do conceito de classe social em estudos epidemiológicos. Rev. Saúde Pública 1988; 22(4):253-265.

9. Kay EJ, Blinkhorn AS. The reasons underlying the extraction of teeth in Scotland. Br Dent J 1986; 160:287-290.
10. Caldas Jr. AF, M arcenes W, Sheiham A. Reasons for tooth extraction in a Brazilian population. Int Dent J 2000; 50: 67-273.

11. Guimarães M M, Marcos B. Perda de dente relacionada a razões clínicas segundo a classe social. Rev CROM G 1995; 1(2):54-61.

12. Gubeissi Filho W, Silva CF, Jorge WA. Avaliação clínica das indicações das exodontias realizadas durante $04^{\circ}$ ano letivo do curso de graduação em Odontologia. Rev Inst ci Saude 1995; 13(1):11-15.

13. Jovino-Silveira RC, Souza EHA, Caldas Júnior AF. Razões para extração de dentes permanentes. Odontol Clin Cienti 2002; 1(3):207-210.

14. Leite $\mathrm{O}, \mathrm{M}$ arcos $\mathrm{B}$, M endes EV. O corrência das exodontias em função da cárie, doença periodontal, indicação protética e outras causas, em Belo Horizonte - Brasil. Arq Cent Est Fac Odontol M G 1975; 12(1/2): 7-30.

15. Machado FA, Godoy HA, Terra AJS, Marzola C. Principais causas das exodontias no município de Campo Grande, M T. Arq Cent Est Fac O dontol M G 1973; 10(1/2):129-151.

16. Barros M BA. A utilização do conceito de classe social nos estudos dos perfis epidemiológicos: uma proposta. Rev. Saúde Pública 1986; 20:269-273.

Artigo apresentado em 27/03/2006

Artigo aprovado em 13/09/2006

Versão final apresentada em 04/10/2006 